\title{
Directional short-time Fourier transform of distributions
}

\author{
Katerina Hadzi-Velkova Saneva* and Sanja Atanasova
}

\author{
${ }^{*}$ Correspondence: \\ saneva@feit.ukim.edu.mk \\ Faculty of Electrical Engineering and \\ Information Technologies, Ss. Cyril \\ and Methodius University, Rugjer \\ Boshkovik bb, Skopje, Macedonia
}

\begin{abstract}
In this paper we consider the directional short-time Fourier transform (DSTFT) that was introduced and investigated in (Giv in J. Math. Anal. Appl. 399:100-107, 2013). We analyze the DSTFT and its transpose on test function spaces $\mathcal{S}\left(\mathbb{R}^{n}\right)$ and $\mathcal{S}\left(\mathbb{Y}^{2 n}\right)$, respectively, and prove the continuity theorems on these spaces. Then the obtained results are used to extend the DSTFT to spaces of distributions.
\end{abstract}

MSC: Primary 44A15; 46F12; secondary 42C20; 44A12; 46F10

Keywords: directional short-time Fourier transform; Radon transform; short-time Fourier transform; distributions

\section{Introduction}

The idea of Candés to study the wavelet theory using directional sensitivity led to the introduction of the ridgelet transform. He introduced and analyzed the continuous ridgelet transform which is a combination of continuous wavelet transform and the Radon transform [2-4]. Motivated by this concept, Grafakos and Sansing [5] introduced a new theory that combines the concept of the Radon transform and time-frequency analysis in a very natural and useful way. The Radon transform and the time-frequency analysis had been separately analyzed for a long time. The Radon transform is a powerful and celebrated tool with many application in astrophysics, computer tomography and seismology. It represents a collection of integrals of a function over all hyperplanes (see equation (2.6)). On the other hand, the short-time Fourier transform (STFT), as a tool of the time-frequency analysis, contains localized time and frequency information of a function. Grafakos and Sansing provided the idea to localize information in time, frequency, and direction defining a directionally sensitive variant of STFT. A slightly different version of their concept was considered by Giv in [1]. He defined the directional short-time Fourier transform (DSTFT) and proved several orthogonality results and reconstruction formulas.

Distribution theory is a very effective device in both pure and applied mathematics and the extension of integral transforms to generalized function spaces is an important research subject. The authors of [6] extended the ridgelet transform on the space of ( $\mathrm{Li}$ zorkin) distributions, while in this paper, we extend the DSTFT introduced by Giv on Schwartz distribution space $\mathcal{S}^{\prime}\left(\mathbb{R}^{n}\right)$. 


\section{Preliminaries}

\subsection{Notation}

The Fourier transform of a function $\phi$ is defined as

$$
\hat{\phi}(w)=\int_{\mathbb{R}^{n}} \phi(x) e^{-2 \pi i x \cdot w} d x .
$$

We use the notations $\mathbb{N}_{0}=\mathbb{N} \cup\{0\}$, and $\mathbb{Y}^{2 n}$ for the set $\mathbb{S}^{n-1} \times \mathbb{R} \times \mathbb{R}^{n}=\{(u, b, a): u \in$ $\left.\mathbb{S}^{n-1}, b \in \mathbb{R}, a \in \mathbb{R}^{n}\right\}$, where $\mathbb{S}^{n-1}$ stands for the unit sphere of $\mathbb{R}^{n}$. We always assume that $n \geq 2$.

For the $L^{2}$ inner product of $f$ and $\phi$ we use $(f, \phi)_{L^{2}}$, while for dual pairing we use $\langle f, \phi\rangle$.

\subsection{Spaces}

The linear space of all rapidly decreasing smooth functions at infinity $\mathcal{S}\left(\mathbb{R}^{n}\right)$ consists of all functions $\phi \in C^{\infty}\left(\mathbb{R}^{n}\right)$ for which

$$
\rho_{\nu}(\phi)=\sup _{x \in \mathbb{R}^{n},|p| \leq \nu}(1+|x|)^{\nu}\left|\phi^{(p)}(x)\right|<\infty, \quad v \in \mathbb{N}_{0}
$$

The dual space of $\mathcal{S}\left(\mathbb{R}^{n}\right)$ is the space of tempered distributions $\mathcal{S}^{\prime}\left(\mathbb{R}^{n}\right)$, [7].

We also need the Lizorkin test function space $\mathcal{S}_{0}\left(\mathbb{R}^{n}\right)$, that is, the space of highly timefrequency localized functions over $\mathbb{R}^{n}[8]$. It is a closed subspace of $\mathcal{S}\left(\mathbb{R}^{n}\right)$, and all moments of an element from $\mathcal{S}_{0}\left(\mathbb{R}^{n}\right)$ are equal to 0 , namely, $\phi \in \mathcal{S}_{0}\left(\mathbb{R}^{n}\right)$ if $\int_{\mathbb{R}^{n}} x^{m} \phi(x) d x=0$, for all $m \in \mathbb{N}_{0}^{n}$.

The space of smooth functions on the sphere is denoted by $\mathcal{D}\left(\mathbb{S}^{n-1}\right)$. Let $\mathcal{A}$ be a locally convex space of smooth test functions on $\mathbb{R} \times \mathbb{R}^{n}$. Then $\mathcal{A}\left(\mathbb{S}^{n-1} \times \mathbb{R} \times \mathbb{R}^{n}\right)$ is the space of functions $\rho(u, b, a)$ that have the properties of $\mathcal{A}$ in the variable $(b, a) \in \mathbb{R} \times \mathbb{R}^{n}$ and being smooth in $u \in \mathbb{S}^{n-1}$. So, we introduce $\mathcal{S}\left(\mathbb{Y}^{2 n}\right)$ as the space of functions $\Phi \in C^{\infty}\left(\mathbb{Y}^{2 n}\right)$ for which

$$
\rho_{s, r}^{l, m, k}(\Phi)=\sup _{(u, b, a) \in \mathbb{Y}^{2 n}}\left(1+|a|^{2}\right)^{s / 2}\left(1+|b|^{2}\right)^{r / 2}\left|\frac{\partial^{l}}{\partial a^{l}} \frac{\partial^{m}}{\partial b^{m}} \Delta_{u}^{k} \Phi(u, b, a)\right|<\infty
$$

for all $l, m, k, s, r \in \mathbb{N}_{0}$. Here, $\Delta_{u}$ stands for the Laplace-Beltrami operator on the unit sphere $\mathbb{S}^{n-1}$. The topology of this space is defined by means of the seminorms (2.2). Its dual space is $\mathcal{S}^{\prime}\left(\mathbb{Y}^{2 n}\right)$, and we will identify a locally integrable function $F$ on $\mathbb{Y}^{2 n}$ with a distribution on $\mathbb{Y}^{2 n}$ as follows. If $F$ satisfies the bound

$$
|F(u, b, a)| \leq C(1+|b|)^{s}(1+|a|)^{s}, \quad(u, b, a) \in \mathbb{Y}^{2 n},
$$

for some $s, C>0$, then $F$ could be identified with an element of $\mathcal{S}^{\prime}\left(\mathbb{Y}^{2 n}\right)$ via

$$
\langle F, \Phi\rangle:=\int_{\mathbb{R}^{n}} \int_{\mathbb{R}} \int_{\mathbb{S}^{n-1}} F(u, b, a) \Phi(u, b, a) d u d b d a, \quad \Phi \in \mathcal{S}\left(\mathbb{Y}^{2 n}\right) .
$$

The nuclearity of the Schwartz spaces [9] yields the equality $\mathcal{S}\left(\mathbb{Y}^{2 n}\right)=\mathcal{D}\left(\mathbb{S}^{n-1}\right) \hat{\otimes} \mathcal{S}(\mathbb{R} \times$ $\mathbb{R}^{n}$ ), where $X \hat{\otimes} Y$ is the topological tensor product space obtained as the completion of $X \otimes Y$ in the $\pi$-topology or the $\varepsilon$-topology [9]. 


\subsection{The STFT}

Let $T_{b} f(\cdot)=f(\cdot-b)$ and $M_{a} f(\cdot)=e^{2 \pi i a \cdot} f(\cdot), a, b \in \mathbb{R}^{n}$ denote the translation and modulation operators, respectively. For the time-frequency shifts $M_{a} T_{b}$ and $T_{b} M_{a}$ the following relation holds:

$$
M_{a} T_{b} f(x)=e^{2 \pi i a \cdot x} T_{b} M_{a} f(x) .
$$

The short-time Fourier transform (STFT) of a function $f \in L^{2}\left(\mathbb{R}^{n}\right)$ with respect to a window function $g \in L^{2}\left(\mathbb{R}^{n}\right)$ is defined as

$$
V_{g} f(b, a)=\left\langle f(x), \overline{M_{a} T_{b} g(x)}\right\rangle_{x}=\int_{\mathbb{R}^{n}} f(x) \overline{g(x-b)} e^{-2 \pi i a \cdot x} d x, \quad b, a \in \mathbb{R}^{n} .
$$

We have $\left\|V_{g} f\right\|_{2}=\|f\|_{2}\|g\|_{2}$. If $\|g\|_{2}=1$, then the STFT is an isometry from $L^{2}\left(\mathbb{R}^{n}\right)$ to $L^{2}\left(\mathbb{R}^{2 n}\right)$. We write $V_{g}^{*}$ for the adjoint of $V_{g}$, given by

$$
V_{g}^{*} F(x)=\iint_{\mathbb{R}^{2 n}} F(b, a) g(x-b) e^{2 \pi i a \cdot x} d a d b .
$$

Let $g \neq 0$ and $\psi \in L^{2}\left(\mathbb{R}^{n}\right)$ be a synthesis window for $g$, where $(g, \psi)_{L^{2}} \neq 0$. Then, for any $f \in L^{2}\left(\mathbb{R}^{n}\right)$,

$$
f=\frac{1}{(\psi, g)_{L^{2}}} \iint_{\mathbb{R}^{2 n}} V_{g} f(b, a) M_{a} T_{b} \psi d a d b
$$

The definition of $V_{g} f$ can be generalized for $f$ in larger classes than $L^{2}\left(\mathbb{R}^{n}\right)$ if the dual pairing in (2.4) is well defined. Whenever $\mathcal{A}\left(\mathbb{R}^{n}\right)$ is a time-frequency shift invariant topological vector space, one can take $g \in \mathcal{A}\left(\mathbb{R}^{n}\right)$ and $f \in \mathcal{A}^{\prime}\left(\mathbb{R}^{n}\right)$, [10]. We emphasize that if $f \in \mathcal{S}\left(\mathbb{R}^{n}\right)$ then equation (2.5) holds pointwise.

\subsection{The Radon transform}

Let $f \in L^{1}\left(\mathbb{R}^{n}\right)$ be a function that is integrable on hyperplanes of $\mathbb{R}^{n}$. The Radon transform of $f$ is defined as

$$
R f(u, p)=R f_{u}(p):=\int_{x \cdot u=p} f(x) d x=\int_{\mathbb{R}^{n}} f(x) \delta(p-x \cdot u) d x
$$

where $\delta$ is the Dirac delta, $u \in \mathbb{S}^{n-1}, p \in \mathbb{R}$, and $x \cdot u=p$ specifies a hyperplane of $\mathbb{R}^{n}$ [11-13]. By Fubini's theorem $R f \in L^{1}\left(\mathbb{S}^{n-1} \times \mathbb{R}\right)$. The Fourier transform and the Radon transform are connected by the Fourier slice theorem. According to it, the Fourier transform of the Radon transform can be computed as

$$
\widehat{\operatorname{Rf}_{u}}(p)=\int_{-\infty}^{\infty} f(x) e^{-2 \pi i x \cdot u p} d x=\widehat{f}(u p), \quad u \in \mathbb{S}^{n-1}, p \in \mathbb{R},
$$

for sufficiently regular $f\left(\right.$ e.g., for $f \in L^{1}\left(\mathbb{R}^{n}\right)$ such that $\left.\widehat{f} \in L^{1}\left(\mathbb{R}^{n}\right)\right)$. 


\subsection{The directional short-time Fourier transform}

Let $g \in \mathcal{S}(\mathbb{R})$ be a non-zero window function and $f \in L^{1}\left(\mathbb{R}^{n}\right)$. As in the case of ridgelets, Grafakos and Sansing in [5] introduced the Gabor ridge functions

$$
g_{u, b, a}(x)=e^{2 \pi i a(u \cdot x-b)} g(u \cdot x-b),
$$

for $(u, b, a) \in \mathbb{Y}^{2 n}$, which can be viewed as time-frequency analysis elements in the Radon domain. By pairing the function $f$ with $g_{u, b, a}$ they have defined a directionally sensitive variant of STFT. Because of the directional modulations of $g_{u, b, a}$, with this transform one can obtain time-frequency information of $f$ in the direction of $u$, while as in the classical time-frequency theory $a$ and $b$ measure the modulation and translation. These authors have shown that it is not possible to obtain an exact reconstruction of a signal using the Gabor ridge functions ([5], Theorem 1), and therefore they have modified their class of functions to the weighted Gabor ridge functions (see [5] for details).

Based on the relation

$$
V_{g} f(b, a)=\widehat{f \cdot T_{b} \bar{g}}(a)
$$

Giv in [1] introduced another transform which is also a directionally sensitive variant of STFT, letting enter of the directional parameter $u$ only in the window $g$, unlike the approach of Grafakos and Sansing. Let $g \in L^{\infty}(\mathbb{R})$ be a non-zero function. For $(u, b, a) \in \mathbb{Y}^{2 n}$, he defined the function $g_{u, b, a}: \mathbb{R}^{n} \rightarrow \mathbb{C}$, as

$$
g_{u, b, a}(x)=e^{2 \pi i x \cdot a} g(x \cdot u-b), \quad x \in \mathbb{R}^{n},
$$

which behaves like a one-dimensional window in the direction of $u$ and is constant along its orthogonal complement, and as in (2.4) the parameter $a$ is used only for taking the Fourier transform.

Giv defines the directional short-time Fourier transform (DSTFT) of an integrable function $f \in L^{1}\left(\mathbb{R}^{n}\right)$ with respect to $g$ via

$$
D S_{g} f(u, b, a):=\int_{\mathbb{R}^{n}} f(x) \overline{g(u \cdot x-b)} e^{-2 \pi i x \cdot a} d x=\left\langle f(x),\left.\bar{g}_{u, b, a}(x)\right|_{x}\right.
$$

where $(u, b, a) \in \mathbb{Y}^{2 n}$. Let we note that with (2.10) we obtain information for the time of the signal along the direction $u$. If we want to obtain information for the time and the frequency of the signal along the direction $u$, we can simply use the relation (2.9).

We must be careful if we want to extend the definition of the directional short-time Fourier transform to more general spaces. Even if $f \in L^{2}\left(\mathbb{R}^{n}\right)$ the defining integral in (2.10) may not converge. Moreover, when trying to extend the directional short-time Fourier transform to distributions, (2.10) is not well defined for $f \in \mathcal{S}^{\prime}\left(\mathbb{R}^{n}\right)$ because $g_{u, b, a} \notin \mathcal{S}\left(\mathbb{R}^{n}\right)$. This problem will be analyzed in Section 3.2 where the directional short-time Fourier transform of tempered distributions for $g \in \mathcal{S}_{0}(\mathbb{R})$ will be defined.

The following lemma gives a useful relation between the directional STFT and the Fourier transform of the function $f$. 
Lemma 2.1 For $g \in \mathcal{S}(\mathbb{R})$ and $f \in L^{1}\left(\mathbb{R}^{n}\right) \cap L^{2}\left(\mathbb{R}^{n}\right)$

$$
D S_{g} f(u, b, a)=\int_{\mathbb{R}}\left(T_{-a} \widehat{f}(u \cdot \omega)\right) M_{-b} \hat{\bar{g}}(\omega) d \omega .
$$

Proof From the definition of the Radon transform, the Fourier slice theorem (2.7), and Plancherel's theorem it is easy to obtain

$$
\begin{aligned}
D S_{g} f(u, b, a) & =\int_{\mathbb{R}} \int_{u \cdot x=s} f\left(t u+s u^{\perp}\right) \overline{g(s-b)} e^{-2 \pi i a\left(t u+s u^{\perp}\right)} d u d s \\
& \left.=\int_{\mathbb{R}} R\left(M_{-a} f\right)_{u}(s) T_{b} \bar{g}(s) d s=\int_{\mathbb{R}} \widehat{R\left(M_{-a} f\right.}\right)_{u}(\omega) \widehat{T_{b} \bar{g}}(\omega) d \omega \\
& =\int_{\mathbb{R}} T_{-a} \hat{f}(u \omega)\left(M_{-b} \hat{\bar{g}}\right)(\omega) d \omega .
\end{aligned}
$$

In [1], Theorem 3.6, it is shown that for non-trivial $g \in \mathcal{S}(\mathbb{R})$ with synthesis window $\psi \in$ $\mathcal{S}(\mathbb{R})$, and $f \in L^{1}\left(\mathbb{R}^{n}\right)$ for which $\hat{f} \in L^{1}\left(\mathbb{R}^{n}\right)$, the following reconstruction formula holds pointwise:

$$
f(x)=\frac{1}{(g, \psi)_{L^{2}}} \int_{\mathbb{R}^{n}} \int_{\mathbb{R}} \int_{\mathbb{S}^{n-1}} D S_{g} f(u, b, a) \psi_{u, b, a}(x) d u d b d a .
$$

Equation (2.12) takes the form $\left(D S_{\psi}^{*} \circ D S_{g}\right) f=(g, \psi)_{L^{2}} f$.

The reconstruction formula (2.12) suggests to define an operator that maps functions on $\mathbb{Y}^{2 n}$ to functions on $\mathbb{R}^{n}$ as superposition of functions $g_{u, b, a}$. Given $g \in \mathcal{S}(\mathbb{R})$, we introduce the directional synthesis operator as

$$
D S_{g}^{*} \Phi(x):=\int_{\mathbb{R}^{n}} \int_{\mathbb{R}} \int_{\mathbb{S}^{n-1}} \Phi(u, b, a) g_{u, b, a}(x) d u d b d a, \quad x \in \mathbb{R}^{n} .
$$

We note that for $\Phi \in \mathcal{S}\left(\mathbb{Y}^{2 n}\right)$, the integral (2.13) is absolutely convergent.

Proposition 2.2 Let $g \in \mathcal{S}(\mathbb{R})$. If $f \in L^{1}\left(\mathbb{R}^{n}\right)$ and $\Phi \in \mathcal{S}\left(\mathbb{Y}^{2 n}\right)$, then

$$
\int_{\mathbb{R}^{n}} f(x) D S_{g}^{*} \Phi(x) d x=\int_{\mathbb{R}} \int_{\mathbb{R}^{n}} \int_{\mathbb{S}^{n-1}} D S_{\bar{g}} f(u, b, a) \Phi(u, b, a) d u d b d a .
$$

We may write (2.14) as

$$
\left\langle f, D S_{\bar{g}}^{*} \Phi\right\rangle=\left\langle D S_{g} f, \Phi\right\rangle
$$

This means that $D S_{\bar{g}}^{*}$ and $D S_{g}$ are formal transposes, and we will follow this idea when defining the distributional directional short-time Fourier transform.

\section{Main results}

\subsection{Continuity of the directional STFT on $\mathcal{S}\left(\mathbb{R}^{n}\right)$}

The aim of this section is to prove that the mapping $D S_{g}: \mathcal{S}\left(\mathbb{R}^{n}\right) \rightarrow \mathcal{S}\left(\mathbb{Y}^{2 n}\right)$ is continuous when $g \in \mathcal{S}(\mathbb{R})$, and $D S_{g}^{*}: \mathcal{S}\left(\mathbb{Y}^{2 n}\right) \rightarrow \mathcal{S}\left(\mathbb{R}^{n}\right)$ is continuous for $g \in \mathcal{S}_{0}(\mathbb{R})$.

For non-trivial $g$, the DSTFT $D S_{g}$ is injective and $D S_{g}^{*}$ is surjective, due to the reconstruction formula (2.12). Recall that we endow $\mathcal{S}\left(\mathbb{Y}^{2 n}\right)$ with the system of seminorms (2.2). 
Notice that we can extend the definition of the DSTFT as a sesquilinear mapping $D S:(f, g) \mapsto D S_{g} f$, whereas the directional synthesis operator extends to the bilinear form $D S^{*}:(\Phi, g) \mapsto D S_{g}^{*} \Phi$.

Theorem 3.1 The mapping $D S: \mathcal{S}\left(\mathbb{R}^{n}\right) \times \mathcal{S}(\mathbb{R}) \rightarrow \mathcal{S}\left(\mathbb{Y}^{2 n}\right)$ is continuous.

Proof We will show that, given $s, r, m, l, k \in \mathbb{N}_{0}$, there exist $\nu, \tau \in \mathbb{N}$ and $C>0$ such that

$$
\rho_{s, r}^{l, m, k}\left(D S_{g} \phi\right) \leq C \rho_{\nu}(\phi) \rho_{\tau}(g), \quad \phi \in \mathcal{S}\left(\mathbb{R}^{n}\right), g \in \mathcal{S}(\mathbb{R})
$$

We may assume that $r$ is even and $s \geq 1$.

1. Using the definition of the directional STFT, we have

$$
\begin{aligned}
& \left|\frac{\partial^{l}}{\partial a^{l}} \frac{\partial^{m}}{\partial b^{m}} \Delta_{u}^{k} D S_{g} \phi(u, b, a)\right| \\
& \quad=\left|\frac{\partial^{l}}{\partial a^{l}} \frac{\partial^{m}}{\partial b^{m}} \sum_{|\alpha|, j \leq 2 k} P_{\alpha, j}(u) \int_{\mathbb{R}^{n}} x^{\alpha} \phi(x) \overline{g^{(j)}(u \cdot x-b)} e^{-2 \pi i a \cdot x} d x\right| \\
& \quad=\left|\sum_{|\alpha|, j \leq 2 k} P_{\alpha, j}(u) \int_{\mathbb{R}^{n}} x^{\alpha} \phi(x) \overline{g^{(j+m)}(u \cdot x-b)}(-1)^{m} e^{-2 \pi i a \cdot x}(-2 \pi i x)^{l} d x\right| \\
& \quad \leq C \sum_{|\alpha|, j \leq 2 k} \int_{\mathbb{R}^{n}} x^{\alpha+l}|\phi(x)|\left|\overline{g^{(j+m)}(u \cdot x-b)}\right| d x,
\end{aligned}
$$

where $P_{\alpha, j}(u)$ are certain bounded polynomials. Setting $\phi_{\alpha+l}(x)=x^{\alpha+l} \phi(x)$, this yields

$$
\rho_{s, r}^{l, m, k}\left(D S_{g} \phi\right) \leq C \sum_{|\alpha|, j \leq 2 k} \rho_{s, r}^{0,0,0}\left(D S_{g(j+m)}\left(\phi_{\alpha+l}\right)\right)
$$

Because multiplication by $x^{\alpha+l}$ and differentiation are continuous operators on $\mathcal{S}$, we can assume that $m=l=k=0$.

2. Observe that, by (2.11),

$$
\begin{aligned}
\left(1+b^{2}\right)^{r / 2} D S_{g} \phi(u, b, a) & =\int_{-\infty}^{\infty} \hat{\phi}(\omega u+a) \overline{\hat{g}}(\omega)\left(1-\frac{\partial^{2}}{\partial \omega^{2}}\right)^{r / 2} e^{-2 \pi i b \omega} d \omega \\
& =\int_{-\infty}^{\infty} e^{-2 \pi i b \omega}\left(1-\frac{\partial^{2}}{\partial \omega^{2}}\right)^{r / 2}(\hat{\phi}(\omega u+a) \overline{\hat{g}}(\omega)) d \omega \\
& =\sum_{|\alpha|, j \leq r} Q_{\alpha, j}(u) \int_{-\infty}^{\infty} e^{-2 \pi i b \omega} \hat{\phi}^{(\alpha)}(\omega u+a) \overline{\hat{g}}^{(j)}(\omega) d \omega
\end{aligned}
$$

for some polynomials $Q_{\alpha, j}$. From (2.11), and writing $g_{j}(x)=x^{j} g(x)$ and also $\phi_{\alpha}(x)=x^{\alpha} \phi(x)$, we conclude that

$$
\rho_{s, r}^{0,0,0}\left(D S_{g} \phi\right) \leq C \sum_{|\alpha|, j \leq r} \rho_{s, 0}^{0,0,0}\left(D S_{g_{j}}\left(\phi_{\alpha}\right)\right)
$$

Consequently, we can assume $r=0$. 
3. Finally, we consider the term that involves multiplication by $\left(1+|a|^{2}\right)^{s / 2}$ in $\rho_{s, 0}^{0,0,0}$.

$$
\begin{aligned}
\left(1+a^{2}\right)^{s / 2}\left|D S_{g} \phi(u, b, a)\right| & =\left|\int_{\mathbb{R}^{n}} \phi(x) \bar{g}(u \cdot x-b)\left(1-\frac{\partial^{2}}{\partial x^{2}}\right)^{s / 2} e^{-2 \pi i a \cdot x} d x\right| \\
& \leq \int_{\mathbb{R}^{n}}\left|e^{-2 \pi i a \cdot x}\left(1-\frac{\partial^{2}}{\partial x^{2}}\right)^{s / 2}(\phi(x) \bar{g}(u \cdot x-b))\right| d x \\
& \leq \sum_{|\alpha|, j \leq s} Q_{\alpha, j}(u) \int_{\mathbb{R}^{n}}\left|\phi^{(\alpha)}(x)\right|\left|\overline{g^{(j)}}(u \cdot x-b)\right| d x \\
& \leq C \rho_{s+2}(\phi) \rho_{s+2}(g) .
\end{aligned}
$$

4. From the previous three estimates, we find that (3.1) holds if we take $v=2 k+l+r+s+2$ and $\tau=2 k+m+r+s+2$.

We now analyze the directional synthesis operator.

Theorem 3.2 The bilinear mapping $D S^{*}: \mathcal{S}\left(\mathbb{Y}^{2 n}\right) \times \mathcal{S}_{0}(\mathbb{R}) \rightarrow \mathcal{S}\left(\mathbb{R}^{n}\right)$ is continuous.

Proof Let $g \in \mathcal{S}_{0}(\mathbb{R}), \Phi \in \mathcal{S}\left(\mathbb{Y}^{2 n}\right)$, and $\phi=D S_{g}^{*} \Phi$. Observe that

$$
\phi(x)=\int_{\mathbb{S}^{n-1}} \int_{\mathbb{R}} \omega^{n-1} e^{-2 \pi i \omega u \cdot x}\left(\int_{\mathbb{R}^{n}} \widehat{\Phi}(u, \omega, a) \frac{\hat{g}(\omega)}{\omega^{n-1}} e^{2 \pi i x \cdot a} d a\right) d \omega d u
$$

hence, by Fourier inversion in polar coordinates,

$$
\hat{\phi}(-\omega u)=\int_{\mathbb{R}^{n}} \widehat{\Phi}(u, \omega, a) \frac{\hat{g}(\omega)}{\omega^{n-1}} e^{2 \pi i x \cdot a} d a,
$$

$\omega \in \mathbb{R}, u \in \mathbb{S}^{n-1}$. In (3.2), $\widehat{\Phi}$ denotes the Fourier transform of $\Phi(u, b, a)$ with respect to the variable $b$.

We now prove the continuity of the bilinear directional synthesis mapping. Since the Fourier transforms $g \mapsto \hat{g}$ and $\Phi \mapsto \widehat{\Phi}$ are continuous automorphisms on the $\mathcal{S}$ spaces, the families (cf. (2.1) and (2.2))

$$
\hat{\rho}_{v}(g)=\rho_{\nu}(\hat{g}), \quad g \in \mathcal{S}_{0}(\mathbb{R}), v=0,1, \ldots
$$

and

$$
\hat{\rho}_{s, r}^{l, m, k}(\Phi)=\rho_{s, r}^{l, m, k}(\widehat{\Phi}), \quad \Phi \in \mathcal{S}\left(\mathbb{Y}^{2 n}\right), l, m, k, s, r \in \mathbb{N}_{0}
$$

are bases of seminorms for the topologies of $\mathcal{S}_{0}(\mathbb{R})$ and $\mathcal{S}\left(\mathbb{Y}^{2 n}\right)$, respectively. We will define a different family of seminorms on $\mathcal{S}\left(\mathbb{R}^{n}\right)$. The seminorms $\dot{\rho}_{N, q, k}$, given by

$$
\dot{\rho}_{N, q, k}(\phi):=\sup _{(u, \omega) \in \mathbb{S}^{n-1} \times \mathbb{R}}\left|\omega^{N} \frac{\partial^{q}}{\partial \omega^{q}} \Delta_{u}^{k} \hat{\phi}(\omega u)\right|, \quad N, q, k \in \mathbb{N}_{0}
$$


are a base of continuous seminorms for the topology of $\mathcal{S}\left(\mathbb{R}^{n}\right)$. We show that given $N, q, k \in$ $\mathbb{N}_{0}$ there is $C>0$ and $v \in \mathbb{N}$ such that

$$
\dot{\rho}_{N, q, k}\left(D S_{g}^{*} \Phi\right) \leq C \hat{\rho}_{n-1+q}(g) \sum_{m \leq v} \hat{\rho}_{2, N}^{0, m, k}(\Phi)
$$

Now, setting again $\phi(x):=D S_{g}^{*} \Phi(x)$, using equation (3.2), the Leibniz formula, and the Taylor expansion for $g$, we get

$$
\begin{aligned}
\left|\omega^{N} \frac{\partial^{q}}{\partial \omega^{q}} \Delta_{u}^{k} \hat{\phi}(\omega u)\right| & \leq C \sum_{j=0}^{q} \sum_{d=0}^{j} \int_{\mathbb{R}^{n}}\left|\omega^{N} \frac{\partial^{q-j}}{\partial \omega^{q-j}} \Delta_{u}^{k} \widehat{\Phi}(u, \omega, a) \frac{\hat{g}^{(j-d)}(\omega)}{\omega^{n-1+d}}\right| d a \\
& =C \sum_{j=0}^{q} \sum_{d=0}^{j} \int_{\mathbb{R}^{n}}\left|\omega^{N} \frac{\partial^{q-j}}{\partial \omega^{q-j}} \Delta_{u}^{k} \widehat{\Phi}(u, \omega, a) \hat{g}^{(j+n-1)}\left(\omega_{0}\right)\right| d a \\
& \leq C \hat{\rho}_{n-1+q}(g) \sum_{j=0}^{q} \hat{\rho}_{2, N}^{0, q-j, k}(\Phi) \int_{\mathbb{R}^{n}} \frac{d a}{a^{2}+1}
\end{aligned}
$$

as claimed.

\subsection{The directional STFT on $\mathcal{S}^{\prime}\left(\mathbb{R}^{n}\right)$}

Using the obtained continuity results, we will define the directional STFT on distributions.

Definition 3.3 Let $g \in \mathcal{S}_{0}(\mathbb{R})$. The directional STFT of $f \in \mathcal{S}^{\prime}\left(\mathbb{R}^{n}\right)$ with respect to $g$ is the element $D S_{g} f \in \mathcal{S}^{\prime}\left(\mathbb{Y}^{2 n}\right)$ whose action on test functions is given by

$$
\left\langle D S_{g} f, \Phi\right\rangle:=\left\langle f, D S_{\bar{g}}^{*} \Phi\right\rangle, \quad \Phi \in \mathcal{S}\left(\mathbb{Y}^{2 n}\right) .
$$

The consistence of Definition 3.3 is guaranteed by Theorem 3.2.

Definition 3.4 Let $g \in \mathcal{S}_{0}(\mathbb{R})$. The directional synthesis operator $D S_{g}^{*}: \mathcal{S}^{\prime}\left(\mathbb{Y}^{2 n}\right) \rightarrow \mathcal{S}^{\prime}\left(\mathbb{R}^{n}\right)$ is defined as

$$
\left\langle D S_{g}^{*} F, \phi\right\rangle:=\left\langle F, D S_{\bar{g}} \phi\right\rangle, \quad F \in \mathcal{S}^{\prime}\left(\mathbb{Y}^{2 n}\right), \phi \in \mathcal{S}\left(\mathbb{R}^{n}\right)
$$

This definition of the directional synthesis operator $D S_{g}^{*}$ for $g \in \mathcal{S}_{0}(\mathbb{R})$ is guaranteed by Theorem 3.1.

The following continuity result is obtained by taking transposes in Theorems 3.1 and 3.2.

Proposition 3.5 Let $g \in \mathcal{S}_{0}(\mathbb{R})$. The directional STFT $D S_{g}: \mathcal{S}^{\prime}\left(\mathbb{R}^{n}\right) \rightarrow \mathcal{S}^{\prime}\left(\mathbb{Y}^{2 n}\right)$ and the directional synthesis operator $D S_{g}^{*}: \mathcal{S}^{\prime}\left(\mathbb{Y}^{2 n}\right) \rightarrow \mathcal{S}^{\prime}\left(\mathbb{R}^{n}\right)$ are continuous linear maps.

Now, we can generalize the reconstruction formula (2.12) on distributions.

Theorem 3.6 (Inversion formula) Let $g \in \mathcal{S}_{0}(\mathbb{R})$ be non-trivial. If $\psi \in \mathcal{S}(\mathbb{R})$ is a synthesis window for $g$, namely, $(\psi, g)_{L^{2}} \neq 0$, then

$$
\operatorname{id}_{\mathcal{S}^{\prime}\left(\mathbb{R}^{n}\right)}=\frac{1}{(g, \psi)_{L^{2}}}\left(D S_{\psi}^{*} \circ D S_{g}\right)
$$


Proof Using Definitions 3.3 and 3.4, and reconstruction formula (2.12), we obtain at once

$$
\left\langle D S_{\psi}^{*}\left(D S_{g} f\right), \phi\right\rangle=\left\langle f, D S_{\bar{g}}^{*}\left(D S_{\bar{\psi}} \phi\right)\right\rangle=(\bar{\psi}, \bar{g})_{L^{2}}\langle f, \phi\rangle=(g, \psi)_{L^{2}}\langle f, \phi\rangle .
$$

\subsection{The directional STFT on $\mathcal{D}_{L^{1}}^{\prime}\left(\mathbb{R}^{n}\right)$}

Here we will employ the Schwartz space $\mathcal{D}_{L^{1}}^{\prime}\left(\mathbb{R}^{n}\right)$, defined in Schwartz' book [7], p.200. It contains all the distributions $f$ which can be represented as

$$
f=\sum_{|\alpha| \leq m} \partial^{\alpha} f_{\alpha}, \quad f_{\alpha} \in L^{1}\left(\mathbb{R}^{n}\right)
$$

for some $m \in \mathbb{N}_{0}$.

It turns out the directional short-time Fourier transform can be canonically defined with (2.10) for distributions $f \in \mathcal{D}_{L^{1}}^{\prime}\left(\mathbb{R}^{n}\right)$, because the test function $g_{u, b, a} \in \mathcal{D}_{L^{\infty}}\left(\mathbb{R}^{n}\right)$ and thus the integral formula can be still interpreted in the sense of Schwartz integrable distributions [7], p.203.

The following theorem shows that Definition 3.3 is consistent with (2.10). Let us note that our definition of the directional STFT for distributions coincides with the one given for the test functions.

Theorem 3.7 Let $f \in \mathcal{D}_{L^{1}}^{\prime}\left(\mathbb{R}^{n}\right)$. Then the directional STFT off can be determined by (2.10), that is,

$$
\left\langle D S_{g} f, \Phi\right\rangle=\int_{\mathbb{R}^{n}} \int_{\mathbb{R}} \int_{\mathbb{S}^{n-1}} D S_{g} f(u, b, a) \Phi(u, b, a) d u d b d a, \quad \Phi \in \mathcal{S}\left(\mathbb{Y}^{2 n}\right) .
$$

Proof Following Schwartz' structural theorem [7], we can write $f=\sum_{j=1}^{N} f_{j}^{\left(m_{j}\right)}$, where each $f_{j} \in L^{1}\left(\mathbb{R}^{n}\right)$. Observe first that from (2.8) and the Leibniz formula

$$
\left\langle f_{j}^{\left(m_{j}\right)}, g_{u, b, a}\right\rangle=(-1)^{m_{j}} \sum_{k=0}^{m_{j}}\left(\begin{array}{c}
m_{j} \\
k
\end{array}\right)\left\langle f_{j}, u^{k}(2 \pi i a)^{m_{j}-k}\left(g^{(k)}\right)_{u, b, a}\right\rangle .
$$

On the other hand, since

$$
(-1)^{\left|m_{j}\right|} \frac{\partial^{\left|m_{j}\right|}}{\partial x^{m_{j}}} D S_{\bar{g}}^{*} \Phi=\sum_{k=0}^{m_{j}}\left(\begin{array}{c}
m_{j} \\
k
\end{array}\right) D S_{\bar{g}^{(k)}}^{*}\left(u^{k}(2 \pi i a)^{m_{j}-k} \Phi\right),
$$

the directional STFT, defined via (3.3), satisfies

$$
D S_{g}\left(f_{j}^{\left(m_{j}\right)}\right)=\sum_{k=0}^{m_{j}}\left(\begin{array}{c}
m_{j} \\
k
\end{array}\right) u^{k}(2 \pi i a)^{m_{j}-k} D S_{g^{(k)}} f_{j} .
$$

Therefore, we may assume that $f \in L^{1}\left(\mathbb{R}^{n}\right)$. But in the latter case, the result is a consequence of Proposition 2.2. 
Authors' contributions

All authors contributed equally to the writing of this paper. All authors read and approved the final manuscript.

Received: 18 December 2015 Accepted: 6 April 2016 Published online: 22 April 2016

\section{References}

1. Giv, HH: Directional short-time Fourier transform. J. Math. Anal. Appl. 399, 100-107 (2013)

2. Candès, EJ: Ridgelet: theory and applications. Ph.D. thesis, Department of Statistics, Stanford University (1998)

3. Candès, EJ: Harmonic analysis of neural networks. Appl. Comput. Harmon. Anal. 6, 197-218 (1999)

4. Candès, EJ, Donoho, DL: Ridgelets: a key to higher-dimensional intermittency? Philos. Trans. R. Soc., Math. Phys. Eng. Sci. 357, 2495-2509 (1999)

5. Grafakos, L, Sansing, C: Gabor frames and directional time-frequency analysis. Appl. Comput. Harmon. Anal. 25(1) 47-67 (2008)

6. Kostadinova, S, Pilipovic, S, Saneva, K, Vindas, J: The ridgelet transform of distributions. Integral Transforms Spec. Funct. 25, 344-358 (2014)

7. Schwartz, L: Théorie des Distributions. Hermann, Paris (1966)

8. Wavelets, HM: An Analysis Tool. The Clarendon Press, Oxford University Press, New York (1995)

9. Trèves, F: Topological Vector Spaces, Distributions and Kernel. Academic Press, New York-London (1967)

10. Gröchenig, K: Foundations of Time-Frequency Analysis. Birkhäuser, Boston (2001)

11. Helgason, S: The Radon Transform, 2nd edn. Birkhäuser, Boston (1999)

12. Ludwig, D: The Radon transform on Euclidean space. Commun. Pure Appl. Math. 19, 49-81 (1966)

13. Ramm, AG: The Radon transform on distributions. Proc. Jpn. Acad., Ser. A, Math. Sci. 71, $202-206$ (1995)

\section{Submit your manuscript to a SpringerOpen ${ }^{\circ}$ journal and benefit from:}

- Convenient online submission

Rigorous peer review

- Immediate publication on acceptance

- Open access: articles freely available online

- High visibility within the field

- Retaining the copyright to your article 